\title{
IMPACTO DE FATORES DE MERCADO NOS RETORNOS DE PREÇOS AGRÍCOLAS
}

\author{
Market Factors Impact on Agricultural Prices Returns
}

\begin{abstract}
RESUMO
Em 2014 os produtos primários representaram quase a metade das exportações brasileiras. O setor agropecuário foi o setor responsável pela maior parte deste resultado, devido principalmente ao aumento da produtividade, a expansão das áreas de cultivo e ao crescimento do consumo doméstico e externo. Apesar do bom desempenho, os produtores do setor agropecuário precisam administrar os riscos que envolvem a produção. Desse modo, este estudo tem como objetivo estimar o risco sistemático de cinco produtos agrícolas mais produzidos no Brasil, açúcar, café, milho, trigo e soja, em relação aos fatores de mercado preço futuro internacional, Índice Bovespa, Índice S\&P 500 e taxa de câmbio. São estimados o modelo de precificação de ativos (CAPM) e a teoria de precificação por arbitragem (APT) com dados diários de janeiro de 2010 a março de 2015. Os resultados mostram que os produtos apresentam sensibilidade positiva aos mercados financeiros e preços futuros e sensibilidade negativa a taxa de câmbio. Isso sugere um comportamento homogêneo dos produtos agrícolas e altamente associado às variações do mercado financeiro e cambial.
\end{abstract}

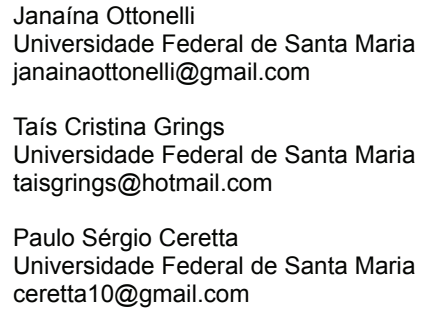

Recebido em: 01/09/2015. Aprovado em: 07/10/2016.

Avaliado pelo sistema double blind review.

Avaliador científico: André Luis Ribeiro Lima

\begin{abstract}
Primary products account for nearly half of Brazil's exports in 2014. The agricultural sector was responsible for most of this, due to the expansion of cultivated areas, and increased productivity and consumption by Asian countries. Despite good performance, the producers from this sector must manage the risks involved in production. The aim of this study was to estimate the systematic risk of more agricultural products produced in Brazil, such as sugar, coffee, corn, wheat and soybeans, in relation to international future price, Bovespa Index, S\&P 500 and the exchange rate. We estimate the asset pricing model (CAPM) and the Arbitrage Pricing Theory (APT) with daily data obtained from January 2010 to March 2015. The results indicate that the products have positive sensitivity to financial markets and future prices and negative sensitivity to exchange rate. The findings suggest that agricultural products have homogeneous behavior and are highly associated to changes in financial and foreign exchange market.
\end{abstract}

Palavras-chave: Produtos Agrícolas, Retorno, Risco Sistemático.

Keywords: Agricultural Products, Return, Systematic Risk.

\section{INTRODUÇÃO}

Nas últimas duas décadas ocorreram grandes mudanças no mercado de produtos agropecuários no Brasil decorrentes do aumento da produtividade, da abertura econômica, do aumento da demanda interna e do grande crescimento no consumo por países populosos como Índia, China e Rússia. Outros fatores que contribuíram para esse cenário foram a expansão das áreas de cultivo nas últimas décadas, a melhoria genética e o desenvolvimento tecnológico e de infraestrutura logística em algumas regiões. A participação dos produtos básicos na exportação brasileira que era de 29,3\% em 2005 passou para 48,7\% em 2014
(BRASIL, 2014). Esse aumento contribuiu positivamente no saldo da balança comercial ao ampliar o interesse do mercado externo na compra dos produtos domésticos.

Apesar do bom desempenho do setor, existem muitos riscos envolvidos no processo de produção e comercialização dos produtos agrícolas que podem estar relacionados a fatores ambientais, climáticos e de crédito. Conforme Geman (2005), a gestão da produção agropecuária envolve a administração frente aos riscos e às alterações na oferta e demanda. A oferta de commodities agropecuárias é composta pelos componentes estoques de produções anteriores, produção do ano corrente e 
importações. A demanda desses produtos corresponde ao uso doméstico e às exportações.

Uma maneira de compreender a sensibilidade dos preços agropecuários aos diferentes fatores de risco consiste em estimar modelos de precificação. Os modelos mais conhecidos são o modelo de precificação de ativos (CAPM) proposto por Sharpe (1964), Lintner (1965) e Mossin (1966) e a teoria de precificação por arbitragem (APT) proposta por Ross (1976). Ambos modelos permitem descobrir a sensibilidade dos ativos a fatores de risco de mercado. O CAPM investiga essa relação para cada ativo em relação a cada fator de mercado considerado isoladamente. A APT investiga a relação de sensibilidade de cada ativo com todos os fatores de mercado conjuntamente.

Estudos que estimam o CAPM para produtos agrícolas indicam uma sensibilidade negativa das commodities a variações no mercado (DUSAK, 1973; GORTON; ROUWENHORST, 2006; DASKALAKI; SKIADOPOULOS, 2011). Outros estudos diferem ao apontar uma sensibilidade positiva das commodities às variações do mercado, sugerindo que as commodities oscilam no mesmo sentido que o mercado (DHUME, 2010; CHEVALLIER; IELPO; BOON, 2013). Com relação a estimação dos riscos pelo modelo APT, existem aplicações para precificação ações (PRIESTLEY, 1996; ANTONIOU; GARRETT; PRIESTLEY, 1998; KRISTJANPOLLER; MORALES, 2011) e não especificamente para os preços dos produtos agropecuários. Ao comparar os dois modelos, CAPM e APT, alguns estudos apontam vantagens em utilizar a APT por permitir uma análise mais completa dos fatores de mercado que influenciam no comportamento dos ativos (MELLO; SAMANEZ, 1999; NEVES; AMARAL, 2002; CALLADO et al., 2010).

Desse modo, este estudo tem como objetivo estimar o risco sistemático dos produtos agrícolas de maior volume de produção no Brasil, que são açúcar, café, milho, trigo e soja, em relação aos fatores de mercado tais como o preço futuro internacional, o Índice Bovespa, o Índice S\&P 500 e a taxa de câmbio. São considerados preços diários em dólares, entre janeiro de 2010 a março de 2015. A estimação dos modelos de precificação pode indicar a quais fatores de mercado os produtos agrícolas apresentam maior sensibilidade.

\section{PRECIFICAÇÃO DE ATIVOS}

Os produtores de commodities enfrentam dificuldades relacionadas a dependência aos fatores climáticos e a volatilidade da oferta, em comparação com uma demanda relativamente estável. Fabozzy, Füss e Kaiser (2008) apontam que a produção de commodities reage de forma lenta às distorções de mercado e, assim, os choques de curto prazo da oferta e demanda são compensados por movimentos nos preços. Os investimentos em commodities diferem dos demais investimentos porque são ativos reais, têm valor intrínseco, fornecem utilidade por uso na fabricação industrial ou no consumo, são ofertados por determinado período (limitado) de ciclo de produção. Outro aspecto importante das commodities como uma classe de ativos é a heterogeneidade.

A produção das commodities é suscetível a volatilidade nos fatores de produção, o que torna mais difícil a intervenção no mercado de tal modo como é possível nos mercados de câmbio e de ações. A volatilidade inerente a essa classe de ativos é a principal razão de muitos investidores evitarem a aplicação de seus recursos em commodities, apesar do fato de que contribuem de forma valiosa na diversificação das carteiras tradicionais devido sua baixa correlação com títulos e ações. Por definição, uma classe de ativos consiste em ativos semelhantes que mostram um perfil homogêneo risco-retorno (a correlação interna de alta), e um perfil de risco-retorno heterogêneo em relação a outras classes de ativos (uma correlação externa baixa) (FABOZZY; FÜSS; KAISER, 2008).

Como a formação dos preços das commodities é resultado da interação entre oferta e demanda em mercados específicos tais produtos não seriam considerados ativos de capital o que tornaria limitada a aplicação do modelo CAPM na precificação (FABOZZY; FÜSS; KAISER, 2008). Mesmo com limitações, muitos estudos estimam o risco sistemático para investigar a sensibilidade das commodities ao mercado por meio do CAPM. Bodie e Rosanski (1980) sugerem que os títulos das commodities podem ser uma atraente classe de investimento porque têm apresentado retorno médio similar ao do S\&P 500.

Gorton e Rouwenhorst (2006) investigaram as commodities como uma classe de ativos considerando o mercado de commodities e o mercado de ações (S\&P 500). Os autores apontam que os retornos das commodities são negativamente correlacionados com o retorno das ações e dos títulos públicos. Esse resultado deve-se em grande parte a diferentes comportamentos no ciclo de negócios, o que indica que as commodities são uma classe de ativos atraente para diversificar carteiras, além de apresentarem menor risco do que o mercado de ações.

Dhume (2010) aplicou o modelo de precificação de ativos para verificar o retorno de contratos futuros de commodities. Os resultados apontam que as commodities 
têm prêmio de risco que variam no tempo. Assim, um choque no mercado de commodities pode não afetar o retorno em outros mercados e contribui para ganhar altos retornos com baixa volatilidade. O risco sistemático pode explicar o retorno das carteiras de commodities, principalmente para as mercadorias como grãos, metal e energia, que ganham retornos significativamente positivos.

Daskalaki e Skiadopoulos (2011) investigaram os benefícios da inclusão das commodities nas carteiras de investimento por meio da estimação dos fatores comuns de risco com o mercado de ações (S\&P 500) e mercado de títulos. Os autores encontraram evidências consistentes de que o desempenho das carteiras que incluem as commodities é inferior a outras classes de ativos. Contudo, indicam os benefícios potenciais da diversificação de carteira com a inclusão de commodities porque verificaram certo grau de heterogeneidade entre as commodities.

Chevallier, Ielpo e Boon (2013) estenderam o estudo de Daskalaki e Skiadopoulos (2011) para determinar o número de fatores comuns de risco a serem incluídos em um modelo de fatores. Os autores mediram os fatores de mercado a partir de informações de ações, taxa de cambio e taxa de juros e concluíram que o risco sistemático do fator de mercado de commodities em relação às commodities é positivo e significativo com alto poder explicativo; para o mercado de câmbio a sensibilidade das commodities é negativa e significativa.

Os estudos que utilizam a teoria de precificação por arbitragem (APT) visam, na sua maioria, investigar a sensibilidade ativos e carteiras de ações negociadas nas bolsas de valores a fatores de mercado. A APT proposta por Ross (1976) assume que os mercados são perfeitamente competitivos e com ausências de distorções. Estabelece que os investidores tem crenças homogêneas com relação ao processo gerador dos retornos, ao qual seria linear e governado por fatores comuns a todos os ativos e que as oportunidades de arbitragem se produzem quando é possível obter um ganho sem aumentar os riscos e os recursos que lhe são atribuídos.

Kude (1998) testou a APT no mercado de capitais brasileiro para quantificar e identificar a natureza econômica das fontes de risco relacionadas à volatilidade do retorno dos ativos. Como variáveis econômicas utilizou o IBOVESPA, Dow Jones Industrial Average, NIKKEI, taxa Overnight, Libor, título de 30 anos do Governo Norte- Americano, M4, Indicador Geral de Produção e Ouro spot Norte-Americano. Inicialmente, aplicou a técnica de análise fatorial sobre a amostra de retornos de ações, verificou a existência de oito fatores fundamentais (fontes de risco) e identificou o IBOVESPA como fonte de risco de mercado.

Mello e Samanez (1999) compararam a performance empírica da APT com o CAPM ao investigar a sensibilidade dos retornos da carteira de investimentos ao indicador de mercado IBOVESPA. Como resultado encontraram que a APT apresenta desempenho superior àquele apresentado pelo modelo CAPM. Utilizando-se o CAPM como aproximação da carteira de mercado um índice como o IBOVESPA, o beta de mercado não é capaz de explicar sozinho o retorno esperado dos ativos. Apontam o modelo APT como a melhor escolha.

Neves e Amaral (2002) analisaram os modelos de precificação de ativos CAPM e APT. No caso do CAPM, utilizaram o IBOVESPA como proxy da carteira de mercado. No caso da APT foram adicionados arbitrariamente dezoito fatores macroeconômicos; Título Público Federal (LBC), Taxa de Juros (CDI), Desemprego, Câmbio, Inflação, Nível de Atividade, Reservas Internacionais, IBOVESPA, Base Monetária (M1), Dow Jones, Nasdaq, Taxa de Juros SELIC, Poupança, Saldo Comercial, Taxa de Juros nos Estados Unidos (Prime Rate), Ouro, Custo de Vida e Salário. Concluíram que a APT apresenta desempenho superior ao CAPM, contudo não descartando a validade deste; apenas constatam que o IBOVESPA quando utilizado sozinho como aproximação da carteira de mercado não é capaz de explicar o retorno esperado dos ativos.

Para instigar a possibilidade de arbitragem entre os retornos das ações das principais empresas de alimentos e bebidas no Brasil, Callado et al. (2010) estimaram a APT utilizando como proxy de mercado os fatores macroeconômicos taxa de inflação, taxa de juros e taxa de câmbio. Dos resultados encontrados, para o período mensal de 2004 a 2007, apenas o retorno das ações de uma empresa de alimentos apresentou sensibilidade significativa estatística e negativamente às variações na taxa de câmbio.

Chen, Roll e Ross (1986), Ibrahim e Aziz (2003) e Fifield, Power e Siclair (2002) argumentam que os preços dos ativos são sensíveis às novidades econômicas. Os preços são influenciados por uma variedade de eventos independentes e alguns eventos possuem maiores efeitos do que outros. A teoria financeira aponta que os investidores podem estimar o risco sistemático, que não é eliminado com a diversificação, dos investimentos em ativos. Contudo não aponta quais são os eventos exógenos econômicos que podem causar essa sensibilidade dos preços. Essa lacuna na teoria permite que o investigador determine as variáveis que possam ter essa relação de influência.

Organizações Rurais \& Agroindustriais, Lavras, v. 18, n. 3, p. 228-237, 2016 


\section{PROCEDIMENTOS METODOLÓGICOS}

Neste estudo buscou-se verificar a sensibilidade dos retornos das commodities domésticas açúcar, café, milho, soja e trigo aos fatores de mercado denominados preço futuro do produto, Índice Bovespa, Índice S\&P 500 e taxa de câmbio. Os preços domésticos estão relacionados a oferta e demanda, interna e externa, e aos preços dos produtos externos. A taxa de câmbio pode influenciar na comercialização, uma vez que a desvalorização cambial tende estimular as exportações domésticas. E os preços também podem ser influenciados por índices de mercado de ações, se houver uma relação dos produtos com o mercado financeiros.

Foram utilizados os preços diários dos produtos agrícolas à vista, em dólares, obtidos da base do Centro de Estudos Avançados em Economia Aplicada (CEPEA, 2015). Os dados referentes aos fatores de mercado foram levantados de duas bases de dados. O preço futuro dos produtos e o Índice S\&P 500 foram obtidos da base internacional Quandl (2015) e o Índice Bovespa e a taxa de câmbio da base do Instituto de Pesquisa Econômica Aplicada (IPEA, 2015).

Os dados diários abrangem o período de 04 de janeiro de 2010 a 31 de março de 2015, totalizando 1177 observações. Decidiu-se por um período mais recente, que abrange os últimos cinco anos. Investiga-se um período posterior aos principais efeitos da crise financeira de 2008 até o período mais próximo para tornar possível a elaboração deste trabalho. Os dados foram transformados em logaritmo natural para obter o retorno diário, que consiste na diferença dos logaritmos $r_{i, t}=\ln \left(P_{i, t}\right)-\ln \left(P_{i, t-1}\right)$.

Os índices de mercado Ibovespa e S\&P 500 foram escolhidos com o objetivo de verificar se os retornos dos produtos agrícolas sofrem influência dos mercados financeiro nacional e internacional. Buscou-se também verificar se os retornos dos preços agrícolas estão associados aos retornos dos preços futuros internacionais respectivos e se são impactados pela taxa de câmbio, uma vez que são produtos que compõem as exportações e contribuem para o bom desempenho do país na balança comercial.

$\mathrm{O}$ investimento em ativos financeiros envolve o retorno esperado e o risco. Conforme Ross et al. (2015), o risco podem ser de dois tipos: i) risco não sistemático, que pode ser eliminado por meio da diversificação; e ii) risco sistemático, que se assume mesmo depois da diversificação, ou seja, é o risco de mercado que influencia a todos os ativos individuais. Desse modo, o que se busca medir é o risco sistemático. A estimação pode ser realizada pelo modelo de precificação de ativos (CAPM) ou pela teoria de precificação por arbitragem (APT). Ambos modelos preveem uma relação positiva entre retorno esperado.

O CAPM foi proposto incialmente por Sharpe (1964) e aperfeiçoado por Lintner (1965) e Mossin (1966). Tem como objetivo investigar a sensibilidade de cada ativo em relação a cada fator de mercado considerado isoladamente. Pode ser estimado por regressão linear simples, conforme a Equação 1 .

$\mathrm{r}_{\mathrm{it}}=\alpha+\beta_{\mathrm{i}} \mathrm{r}_{\mathrm{M}, \mathrm{t}}+\varepsilon_{\mathrm{i}}$

em que é o retorno esperado de cada uma das commodities $i$, são elas: açúcar, café, milho, soja e trigo; $t$ é o tempo diário de 04 de janeiro de 2010 a 31 de março de 2015; é a constante ou intercepto; é o coeficiente de variância sistemática ou sensibilidade do retorno das commodities $i$ ao retorno do mercado; é o retorno do portfólio mercado $M$, neste estudo são considerados separadamente o preço futuro, a taxa de câmbio, o Índice Bovespa e o Índice S\&P 500; é o erro aleatório.

O parâmetro $\beta_{i}$ mede a sensibilidade do retorno das commodities às mudanças do mercado. Um valor de $\beta_{1}>1$ sugere que as variações de preço das commodities tende a ampliar as mudanças que ocorrem no mercado de capitais, o que pode ser considerado como investimento agressivo, enquanto que commodities que tem $\beta_{i}<1$ são menos sensíveis às variações de mercado, sendo alternativa conservadora de investimento.

A teoria de precificação por arbitragem (APT) foi proposta por Ross (1976) e pressupõe que os retornos dos títulos são gerados por um conjunto de fatores de mercado. Tem como objetivo investigar a relação de sensibilidade de cada ativo com todos os fatores de mercado conjuntamente, ou seja, em um mesmo modelo são estimados múltiplos riscos que podem afetar a precificação do ativo. Com relação ao CAPM, a APT apresenta um grau de explicação grau de explicação geral $\left(R^{2}\right)$ para a estimação como um todo e como vantagem apresenta a capacidade de lidar com diversos fatores no mesmo modelo, conforme mostra a Equação 2:

$$
\mathrm{r}_{\mathrm{it}}=\alpha+\beta_{1} \mathrm{r}_{\mathrm{PF}, \mathrm{t}}+\beta_{2} \mathrm{r}_{\mathrm{IB}, \mathrm{t}}+\beta_{3} \mathrm{r}_{\mathrm{SP}, \mathrm{t}}+\beta_{4} \mathrm{r}_{\mathrm{TC}, \mathrm{t}}+\varepsilon_{\mathrm{i}}
$$

em que é o retorno esperado de cada uma das commodities $i$ é influenciado conjuntamente pelos fatores de mercado: retorno do preço futuro da commodity considerada, retorno do Índice Bovespa, retorno do Índice S\&P 500 
e retorno da taxa de câmbio; os betas representam a sensibilidade do retorno das commodities em relação a cada fator de mercado; $t$ é o tempo diário de 04 de janeiro de 2010 a 31 de março de 2015; é a constante ou intercepto; é o erro aleatório.

O Índice Bovespa representa as influências do mercado financeiro doméstico. Já os fatores preço futuro do produto no mercado externo, Índice S\&P 500 e a taxa de câmbio investigam a influência dos mercados externos nos preços dos produtos internos. Portanto, busca-se verificar a sensibilidade do retorno dos preços dos produtos agrícolas brasileiros diante de fatores de mercado doméstico e internacional por meio da estimação dos modelos, realizada no software livre Gretl 1.9.14 (2014).

\section{RESULTADOS E DISCUSSÃO}

A Tabela 1 apresenta as estatísticas descritivas dos retornos das variáveis consideradas no período diário de 04 de janeiro de 2010 a 31 de março de 2015. A apresentação dos dados na tabela está dividida em três partes. A parte superior corresponde aos retornos dos preços à vista dos ativos individuais das commodities brasileiras. As demais partes contemplam os fatores de mercado considerados no estudo, sendo que a parte intermediária corresponde aos retornos dos preços futuros dos produtos agrícolas internacionais e a parte inferior aos demais indicadores de mercado Índice Bovespa, Índice S\&P 500 e taxa de câmbio.

Os retornos dos preços agrícolas individuais apresentam retorno médio negativo ao longo do tempo, com a maior queda verificada para o açúcar e maior suba para o café. O produto que apresentou maior desvio padrão foi o café. Com relação aos produtos agrícolas internacionais, também se verificou um retorno médio negativo para todos os produtos, em que o milho apresentou maior queda e o açúcar maior suba. Os produtos que apresentaram maior variabilidade dos preços foram açúcar, seguido de café, trigo e milho. Nota-se, pelo desvio padrão, uma maior variabilidade dos preços futuros internacionais em relação aos produtos à vista domésticos. No que diz respeito a assimetria e excesso de curtose constata-se para ambos produtos agrícolas doméstico e internacional a presença de caudas grossas (fat tail).

Para verificar a presença de raiz unitária e testar a estacionariedade dos dados utilizou-se de dois testes. O teste ADF-GLS proposto por Elliot, Rothenberg e Stock (1996) que testa a hipótese nula de que os retornos tem

TABELA 1 - Estatísticas descritivas dos retornos diários correspondente aos ativos individuais e indicadores de mercado

\begin{tabular}{|c|c|c|c|c|c|c|}
\hline Variáveis & Média & Mínimo & Máximo & Desvio Padrão & Assimetria & Ex. Curtose \\
\hline \multicolumn{7}{|c|}{ Commodities brasileiras } \\
\hline Açúcar & $-0,0007$ & $-0,124$ & 0,054 & 0,012 & $-1,030$ & 10,997 \\
\hline Café & $-0,0001$ & $-0,093$ & 0,108 & 0,021 & 0,121 & 2,276 \\
\hline Milho & $-0,0002$ & $-0,060$ & 0,072 & 0,013 & 0,514 & 3,274 \\
\hline Soja & $-0,0001$ & $-0,047$ & 0,049 & 0,012 & $-0,124$ & 1,102 \\
\hline Trigo & $-0,0002$ & $-0,042$ & 0,065 & 0,012 & 0,276 & 1,905 \\
\hline \multicolumn{7}{|c|}{ Commodities internacionais } \\
\hline Açúcar & $-0,0007$ & $-0,183$ & 0,166 & 0,024 & $-0,288$ & 7,469 \\
\hline Café & $-0,0001$ & $-0,114$ & 0,118 & 0,023 & 0,419 & 2,691 \\
\hline Milho & $-0,0001$ & $-0,269$ & 0,133 & 0,021 & $-1,739$ & 25,876 \\
\hline Soja & $-0,0001$ & $-0,141$ & 0,073 & 0,016 & $-1,192$ & 8,241 \\
\hline Trigo & $-0,0001$ & $-0,092$ & 0,107 & 0,022 & 0,200 & 2,351 \\
\hline \multicolumn{7}{|c|}{ Outros indicadores de mercado } \\
\hline Índice Bovespa & $-0,0003$ & $-0,084$ & 0,050 & 0,015 & $-0,171$ & 1,386 \\
\hline Índice S\&P 500 & 0,0005 & $-0,069$ & 0,046 & 0,011 & $-0,440$ & 4,628 \\
\hline Taxa de câmbio & 0,0005 & $-0,034$ & 0,047 & 0,008 & 0,518 & 3,728 \\
\hline
\end{tabular}

Fonte: Resultados do estudo 
raiz unitária, ou seja, não são estacionários. E o teste KPSS proposto por Kwiatkowski et al. (1992) que testa a hipótese nula de que não existe raiz unitária e, portanto, os retornos são estacionários. Conforme os resultados da Tabela 2 os resultados do teste ADF-GLS rejeitam a hipótese nula indicando a ausência de raiz unitária. Os resultados do teste KPSS não rejeitam a hipótese nula, confirmando a estacionariedade das séries de retornos.

Investigou-se também a existência de correlação entre as variáveis consideradas. Entre os índices de mercado financeiro IBOVESPA e S\&P500 há uma correlação de 0,6 e destes índices de mercado com a taxa de câmbio a relação fica em torno do valor negativo de 0,3 , o que indica baixa relação entre os fatores de mercado. A Tabela 3 mostra a correlação de cada produto com seu valor futuro, IBOVESPA, S\&P 500 e taxa de câmbio. Os produtos que apresentam maior correlação com o valor futuro são açúcar, café e soja. Os que apresentam maior relação com os mercados financeiros foram trigo, soja e milho. Todos os produtos apresentam relação negativa com a taxa de câmbio, com destaque para o trigo e milho.

TABELA 2 - Testes de raiz unitária

\begin{tabular}{|c|c|c|c|c|}
\hline \multirow{2}{*}{ Variáveis } & \multicolumn{2}{|c|}{ ADF-GLS } & \multicolumn{2}{|c|}{ KPSS } \\
\hline & Estatística & p-valor & Estatística & p-valor \\
\hline \multicolumn{5}{|c|}{ Commodities Brasileiras } \\
\hline Açúcar & $-1,963$ & 0,05 & 0,089 & $>0,10$ \\
\hline Café & $-7,534$ & 0,00 & 0,236 & $>0,10$ \\
\hline Milho & $-2,922$ & 0,00 & 0,283 & $>0,10$ \\
\hline Soja & $-1,595$ & 0,10 & 0,266 & $>0,10$ \\
\hline Trigo & $-4,257$ & 0,00 & 0,487 & $>0,05$ \\
\hline \multicolumn{5}{|c|}{ Commodities Internacionais } \\
\hline Açúcar & $-3,183$ & 0,00 & 0,076 & $>0,10$ \\
\hline Café & $-12,718$ & 0,00 & 0,186 & $>0,10$ \\
\hline Milho & $-8,210$ & 0,00 & 0,307 & $>0,10$ \\
\hline Soja & $-11,083$ & 0,00 & 0,289 & $>0,10$ \\
\hline Trigo & $-4,889$ & 0,00 & 0,119 & $>0,10$ \\
\hline \multicolumn{5}{|c|}{$\begin{array}{l}\text { Outros indicadores de } \\
\text { mercado }\end{array}$} \\
\hline Índice Bovespa & $-5,814$ & 0,00 & 0,026 & $>0,10$ \\
\hline Índice S\&P 500 & $-9,673$ & 0,00 & 0,041 & $>0,10$ \\
\hline Taxa de câmbio & $-6,014$ & 0,00 & 0,365 & $>0,10$ \\
\hline
\end{tabular}

Fonte: Resultados do estudo.

TABELA 3 - Correlação dos produtos agrícolas com os fatores de mercado

\begin{tabular}{ccccc}
\hline Produtos & Preço Futuro & IBOVESPA & S\&P500 & Taxa de Câmbio \\
\hline Açúcar & 0,912 & $-0,002$ & 0,011 & $-0,057$ \\
Café & 0,758 & 0,225 & 0,212 & $-0,322$ \\
Milho & 0,138 & 0,291 & 0,309 & $-0,462$ \\
Soja & 0,446 & 0,314 & 0,342 & $-0,323$ \\
Trigo & 0,089 & 0,361 & 0,340 & $-0,529$ \\
\hline
\end{tabular}

Fonte: Resultados do estudo 
A Tabela 4 apresenta os resultados da estimação do risco sistemático pelo CAPM para o retorno dos preços de cada produto agrícola brasileiro em relação a cada fator de mercado. Todos os produtos apresentaram sensibilidade positiva e significativa ao preço futuro internacional. Destacase a sensibilidade dos produtos café e soja para os quais as variações do preço externo apresentam grau de explicação $R^{2}$ de $57 \%$ e $19 \%$ na variação nos preços, respectivamente.

Com relação aos índices Bovespa e S\&P 500 os produtos também apresentaram sensibilidade positiva e significativa. No entanto, a sensibilidade foi maior em relação ao índice do mercado financeiro internacional do que ao índice de mercado financeiro doméstico. As sensibilidades dos produtos açúcar, milho, soja e trigo apresentam grau de explicação entre $8 \%$ e $13 \%$ das variações nos índices. Mais uma vez o café foi o produto que apresentou maior sensibilidade, em que $57 \%$ das variações nos preços são explicados pelas variações no IBOVESPA e 4,5\% pelas varrições no S\&P 500 .

E com relação a taxa de câmbio os produtos apresentaram sensibilidade negativa e significativa, com maior impacto nos produtos café e açúcar. Os produtos açúcar, trigo e milho apresentam maior sensibilidade à taxa, que apresenta um grau de explicação de, respectivamente, $28 \%$, $27 \%$ e $21 \%$ nas variações nos preços. A relação negativa indica um comportamento de preços em sentidos opostos, ou seja, uma elevação da taxa de câmbio leva a uma queda no retorno dos produtos agrícolas.

Na Tabela 5 são apresentados os resultados da estimação do risco sistemático pela teoria de precificação por arbitragem (APT). Para o retorno dos preços de cada produto agrícola doméstico estimou-se um modelo para verificação conjunta dos fatores de mercado. Com relação à variação do preço futuro, os produtos açúcar, café, milho e soja apresentaram sensibilidade positiva e significativa. O produto trigo não apresentou sensibilidade à variação dos preços futuros.

Com relação aos indicadores de mercado financeiro, os produtos apresentaram sensibilidade positiva e significativamente. Assim como encontrado no CAPM, pela estimação da APT os produtos também foram mais sensíveis ao mercado financeiro internacional do que ao doméstico. E, com relação à taxa de câmbio, os produtos também apresentam sensibilidade negativa e significativa, com destaque para os produtos açúcar, trigo e milho.

TABELA 4 - Resultados da estimação do CAPM

\begin{tabular}{ccccccccc}
\hline \multirow{2}{*}{ Variáveis } & \multicolumn{2}{c}{ Preço Futuro } & \multicolumn{2}{c}{ IBOVESPA } & \multicolumn{2}{c}{ S\&P 500 } & \multicolumn{2}{c}{ Taxa de câmbio } \\
\cline { 2 - 9 } & $\beta$ & $R^{2}(\%)$ & $\beta$ & $R^{2}(\%)$ & $\beta$ & $R^{2}(\%)$ & $\beta$ & $R^{2}(\%)$ \\
\hline Açúcar & $0,115^{*}$ & 5,05 & $0,278^{*}$ & 11,43 & $0,395^{*}$ & 11,52 & $-0,802 *$ & 28,21 \\
Café & $0,705^{*}$ & 57,40 & $0,318^{*}$ & 57,40 & $0,425^{*}$ & 4,50 & $-0,837^{*}$ & 4,50 \\
Milho & $0,086^{*}$ & 1,91 & $0,252^{*}$ & 8,48 & $0,377^{*}$ & 9,53 & $-0,735^{*}$ & 21,42 \\
Soja & $0,322^{*}$ & 19,89 & $0,244^{*}$ & 9,88 & $0,376^{*}$ & 11,69 & $-0,462^{*}$ & 10,45 \\
Trigo & $0,048^{*}$ & 0,79 & $0,282^{*}$ & 13,01 & $0,376^{*}$ & 11,58 & $-0,760^{*}$ & 27,97 \\
\hline
\end{tabular}

Nota: * p-valor $<0,05$;

Fonte: Resultados da estimação

TABELA 5 - Resultados da estimação da APT

\begin{tabular}{lccccc}
\hline \multicolumn{1}{c}{ Variáveis } & Preço Futuro & IBOVESPA & S\&P 500 & Taxa de Câmbio & \multirow{2}{*}{$R^{2}(\%)$} \\
\hline Açúcar & $\beta_{1}$ & $\beta_{2}$ & $\beta_{3}$ & $\beta_{4}$ & \\
Café & $0,060^{*}$ & $0,093^{*}$ & $0,132^{*}$ & $-0,662^{*}$ & 33,61 \\
Milho & $0,666^{*}$ & 0,060 & $0,103^{*}$ & $-0,399^{*}$ & 61,20 \\
Soja & $0,047^{*}$ & $0,073^{*}$ & $0,151^{*}$ & $-0,618^{*}$ & 25,33 \\
Trigo & $0,265^{*}$ & $0,090^{*}$ & $0,167^{*}$ & $-0,235^{*}$ & 30,52 \\
\hline
\end{tabular}

Nota: * p-valor $<0,05 ; * *$ p-valor $<0,10$;

Fonte: Resultados da estimação

Organizações Rurais \& Agroindustriais, Lavras, v. 18, n. 3, p. 228-237, 2016 
O grau de explicação, $R^{2}$, das variações dos produtos domésticos em relação às variações dos indicadores de mercado foram maiores na APT do que no CAPM. Destaca-se o café em que $61 \%$ das variações são explicadas pelos indicadores de mercado, seguido pelo açúcar com $33 \%$, trigo com $32 \%$ e soja com $32 \%$.

Quando comparados os resultados da estimação dos modelos CAPM e APT, o CAPM indica que os fatores de mercado explicam pouco as variações dos ativos isoladamente. Com exceção do café, que apresentou alto grau de explicação para o valor futuro e IBOVESPA (57\%), os resultados apontam valores baixos de grau de explicação entre as variáveis. O grau de explicação varia entre $0,7 \%$ e $19,8 \%$ considerando o valor futuro, entre $8 \%$ e $13 \%$ para o IBOVESPA, entre $4,5 \%$ e $11,6 \%$ para o S\&P 500 e entre $4 \%$ e $28 \%$ para a taxa de câmbio. Os resultados da estimação da APT indicam grau de explicação dos fatores de mercado em conjunto mais elevado, variando entre $25 \%$ e $33 \%$ nos produtos considerados.

Os preços dos produtos agrícolas domésticos apresentaram resultados semelhantes quando comparados, com relação positiva e significativa com preço futuro, IBOVESPA e S\&P 500, com exceção do trigo. A relação positiva com mercado de ações difere do que foi encontrado por Gorton e Rouwenhorst (2006). E relação negativa e significativa com a taxa de câmbio, o que está de acordo com Chevallier, Ielpo e Boon (2013) e Callado et al. (2010). Isso indica que variações nos fatores de mercado podem ser consideradas responsáveis por parte das variações nos preços dos produtos domésticos.

De modo geral, os resultados apontam homogeneidade do comportamento dos preços frente aos fatores de mercado, com alta correlação com o mercado financeiro, o que difere do que foi apontado por Fabozzy, Füss e Kaiser (2008) e Daskalaki e Skiadopoulos (2011). No Brasil, o mercado de commodities ainda é bastante tradicional, com formação de preços baseada na oferta e demanda. A comercialização de ativos das commodities no mercado financeiro ainda é incipiente e, por apresentarem relação com os fatores de mercado, podem não ser a melhor opção na diversificação de carteira de investimentos.

\section{CONSIDERAÇÕES FINAIS}

Este trabalho teve como objetivo verificar o risco sistemático das commodities agrícolas arroz, café, milho, soja e trigo aos fatores de mercado preço futuro, IBOVESPA, S\&P 500 e taxa de câmbio, no período diário de janeiro de 2010 a março de 2015. Por meio da estimação do CAPM e APT foi possível verificar a relação das variáveis aos fatores de mercado de forma individual e conjunta, respectivamente.

Os resultados do CAPM permitem verificar a sensibilidade dos preços a cada fator de mercado. Indicam que os produtos que apresentam maior grau de associação: ao preço futuro são café e soja; ao IBOVESPA o café, trigo e açúcar; ao S\&P 500 o trigo, açúcar e soja; e a taxa de câmbio o trigo, açúcar e milho. Os resultados da APT indicam que os fatores de mercado considerados na estimação explicam conjuntamente dois terços das variações no preço do café, um terço da variação do açúcar e trigo e um quarto da variação do milho.

As commodities domésticas apresentam comportamento semelhante com relação aos fatores de mercado considerados. A sensibilidade positiva aos mercados financeiros e preços futuros e negativa a taxa de câmbio indica um comportamento homogêneo e altamente associado às variações do mercado financeiro e cambial. Portanto, o efeito das commodities brasileiras na diversificação de carteiras é muito limitado. Esse resultado com preços domésticos à vista difere das pesquisas empíricas anteriores encontradas para preços internacionais futuros. Isso indica a pouca participação dos produtos agrícolas brasileiros no mercado financeiro.

\section{REFERÊNCIAS}

ANTONIOU, A.; GARRETT, I.; PRIESTlEY, R. Macroeconomic variables as common pervasive risk factors and the empirical content of the arbitrage pricing theory. Journal of Empirical Finance, v. 5, p. 221-240, 1998.

BODIE, Z.; ROSANSKY, V. Risk and Return in Commodity Futures. Financial Analysts Journal, v. 36, n. 3, p. 27-39, 1980.

BRASIL. Ministério do Desenvolvimento, Indústria e Comércio Exterior. Balança comercial Brasileira: dados consolidados. Brasília, DF, 2014. Disponível em: http://www.mdic.gov.br. Acesso em 02 mar. 2015 . 
CALLADO, A. A. C.; CALLADO, A. L. C.; MÖLLER, H. D.; LEITÃO, C. R. S. Relações entre os retornos das ações e variáveis macroeconômicas: um estudo entre empresas do setor de alimentos e bebidas através do modelo APT. Sociedade, Contabilidade e Gestão, v. 5, p. 6- 18, jan./ jun. 2010.

CENTRO DE ESTUDOS AVANÇADOS EM ECONOMIA APLICADA DA ESALQ/USP -CEPEA. Indicadores de preços agropecuários. Disponível em: www.cepea.esalq. usp. Acesso: 15 abr. 2015.

CHEN, N-F.; ROLL, R.; ROSS, S. Economic Forces and the Stock Market. The Journal of Business, v. 59, p. 383-403, 1986.

CHEVALLIER, J., IELPO, F., BOON, L-N. Common risk factors in commodities. Economics Bulletin, v. 33, n. 4, p. 2801-2816, 2013.

DASKLAKI, C., SKIADOPOULOS, G. Should Investors Include Commodities in their Portfolios After All? Journal of Banking and Finance, v. 35, n. 10, p. 2606-2626, 2011.

DHUME, D. Using durable consumption risk to explain commodities returns. Working paper, Harvard University, 2010.

DUSAK, K. Futures Trading and Investor Returns: An Investigation of Commodity Market Risk Premiums. Journal of Political Economy, v. 81, n. 6, p. 1387-1406, 1973.

ELLIOTT, G.; ROTHENBERG, T. J.; STOCK, J. H. Efficient tests for an autoregressive unit root. Econometrica, v. 64, n. 04, p. 813-836, 1996.

FABOZZI, F. J.; FÜSS, R.; KAISER, D. G. A Primer on Commodity Investing. In: FABOZZI, F. J.; FÜSS, R.; KAISER, D. G. The Handbook of Commodity Investing. New Jersey: John Wiley \& Songs, 2008.

FIFIELD, S. G. M; POWER, D.M; SINCLAIR, C. D. Macroeconomic factors and share returns: An analysis using emerging market data. International Journal of Finance \& Economics, v. 7, n. 1, p. 51-62, 2002.

GEMAN, H. Commodities and Commodity Derivatives: modeling and pricing for agriculturals, metals and energy. Chichester: Wiley, 2005.
GORTON, G. B.; ROUWENHORST, G. K. Facts and fantasies about commodity futures. Financial Analysts Journal, v. 62, 47-68, 2006.

GRETL. General Public Licence (GNU) Regression, Econometrics and Time-series Library. Disponível em: http://gretl.sourceforge.net/pt.html. Acesso: 31 mar. 2014.

IBRAHIM, M. H; AZIZ, H. Macroeconomic variables and the Malaysian equity market: A view through rolling samples. Journal of Economic Studies, v. 30, n. 1, p. 6-27, 2003.

INSTITUTO DE PESQUISAECONÔMICAAPLICADA. IPEADATA, 2015. Disponível em: http://www.ipea.gov. br. Acesso: 20 abr. 2015.

KRISTJANPOLLER, W.; MORALES, M. Teoría de la asignación de precio por arbitraje aplicada al mercado accionario chileno. Lectures de Economía, n. 74, p. 35-59, 2011.

KUDE, B. A precificação de ativos através da arbitrage pricing theory no mercado de capitais brasileiro. In: ENCONTRO DA ASSOCIAÇÃO NACIONAL DE PROGRAMAS DE PESQUISA EM ADMINISTRAÇÃO (ENANPAD), Foz do Iguaçu, 22., 1998.

KWIATKOWSKI, D.; PHILLIPS P. C. B.; SCHMIDT, P.; SHIN, Y.1992. Testing the null of stationarity against the alternative of a unit root: How sure are we that economic time series have a unit root? Journal of Econometrics, v. 54, p.159-178, 1992.

LINTNER, J. The Valuation of Risk Assets and the Selection of Risky Investments in Stock Portfolios and Capital Budgets. The Review of Economics and Statistics, v. 47, n. 1, p. 13-37, 1965.

MELLO, L. M; SAMANEZ, C. P. Determinação e análise de desempenho do modelo APT - arbitrage pricing theory - no mercado de capitais brasileiro. In: ENCONTRO DA ASSOCIAÇÃO NACIONAL DE PROGRAMAS DE PESQUISA EM ADMINISTRAÇÃO (ENANPAD), 23., Foz do Iguaçu, 1999.

MOSSIN, J. Equilibrium in a Capital Asset Market. Econometrica, v. 34, n. 4, p. 768-783, 1966.

Organizações Rurais \& Agroindustriais, Lavras, v. 18, n. 3, p. 228-237, 2016 
NEVES, A. W.; AMARAL, H. F. A Precificação de Ativos de Renda Variável no Mercado de Capitais Brasileiro: Uma Visão Comparativa entre a Arbitrage Pricing Theory e o Capital Asset Pricing Model, In: ENCONTRO DA ASSOCIAÇÃO NACIONAL DE PROGRAMAS DE PESQUISA EM ADMINISTRAÇÃO (ENANPAD), 26., Salvador, 2002.

PRIESTLEY, R. The arbitrage pricing theory, macroeconomic and financial factors, and expectations generating process. Journal of Banking \& Finance, v. 20, p. 869-890, 1996.
QUANDL. Data Platform. Disponível em: www.quandl. com. Acesso: 15 abr. 2015.

ROSS, S. A. The arbitrage theory of capital asset pricing. Journal of Economic Theory, v. 13, p. 241-360, 1976.

ROSS, S. A.; WESTERFIELD, R. W.; JAFFE, J. F; LAMB, R. Administração Financeira: versão brasileira de corporate finance. 10 ed. Porto Alegre: AMGH, 2015.

SHARPE, W. L. Capital Asset Prices: A Theory of Market Equilibrium under Conditions of Risk. The Journal of Finance, v. 19, n. 3, p. 425-442, 1964. 